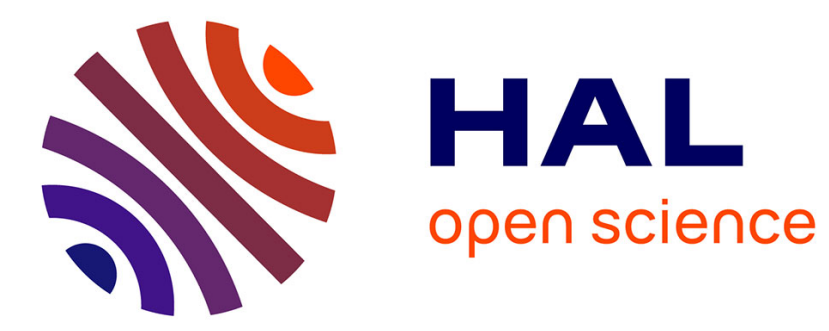

\title{
Persistent slip bands: The bowing and passing model revisited
}

Ladislas Kubin, Maxime Sauzay

\section{To cite this version:}

Ladislas Kubin, Maxime Sauzay. Persistent slip bands: The bowing and passing model revisited. Acta Materialia, 2017, 132, pp.517-524. 10.1016/j.actamat.2017.04.064 . hal-01629111

\section{HAL Id: hal-01629111 \\ https://hal.science/hal-01629111}

Submitted on 5 Jul 2021

HAL is a multi-disciplinary open access archive for the deposit and dissemination of scientific research documents, whether they are published or not. The documents may come from teaching and research institutions in France or abroad, or from public or private research centers.
L'archive ouverte pluridisciplinaire HAL, est destinée au dépôt et à la diffusion de documents scientifiques de niveau recherche, publiés ou non, émanant des établissements d'enseignement et de recherche français ou étrangers, des laboratoires publics ou privés. 
Persistent slip bands: The bowing and passing model revisited

\author{
Ladislas Kubin $^{\mathrm{a}^{*}}$ and Maxime Sauzay ${ }^{\mathrm{b}}$ \\ a Laboratoire d'Étude des Microstructures, CNRS-ONERA (UMR 104 CNRS), \\ 92322 Châtillon Cedex, France \\ ${ }^{\mathrm{b}}$ Commissariat à l'Énergie Atomique, DEN-DMN-SRMA, Bât. 455, \\ 91191 Gif-sur-Yvette Cedex, France
}

\begin{abstract}
Brown's bowing and passing model for persistent slip bands (PSBs) was extended in $\mathrm{Cu}$ and Ni to the whole range of temperatures in which a saturation plateau is observed. Advantage was taken of the similitude relation to rewrite in dimensionless form the equations of the original model and of a more accurate revisited version. Input quantities for computing the solutions were taken from a previous study of experimental results; all unknown quantities could then be directly calculated without any assumption or approximation. The comparison between experimental results and the predictions of the revisited model confirms the basic assumptions of the bowing and passing model, according to which the thermally activated annihilation of screw dipoles is governing the channel widths, the Orowan stresses and the critical stresses in the channels. All other assumptions and numerical predictions are perfectly confirmed, save for the occurrence of small resistive stresses in the channels. In addition, a better understanding of the complex behavior of PSB walls under stress is necessary in order to accurately determine the plastic strain amplitude of PSBs. Mesoscale and atomistic simulations are needed for further modeling of the wall properties and the screw dipole annihilations.
\end{abstract}

Keywords: Persistent slip bands, Saturation stress, Similitude, Modeling, Screw annihilations.

\footnotetext{
*Corresponding author. E-mail addresses: ladislas.kubin@onera.fr (L.P. Kubin), maxime.sauzay@cea.fr (Maxime Sauzay).
} 


\section{Introduction}

Persistent slip bands (PSBs) are formed in ductile materials cycled in single slip at imposed plastic strain amplitudes per cycle typically between $10^{-4}$ and $10^{-2}$. Since their discovery in the 1950s these localized bands of plastic strain have drawn a lot of attention for practical reasons related to fatigue damage as well as for fundamental reasons. Indeed, they constitute a unique example of mechanical response exhibiting a constant saturation stress. Hence, there is a wealth of experimental and theoretical literature on the relation between their almost periodic wall-and-channel dislocation structure and their properties [1-4].

The PSB channels contain a constant density of screw dislocations, which shuttle forward and backward during cycling. These dislocations mutually annihilate by cross-slip after having travelled a certain mean free path and deposited edge segments along the PSB walls. A small fraction of these edge segments is able to cross the walls; it emerges and expands in the neighboring channels where it produces fresh screw dislocations of both signs. Hence, the saturation of the screw dislocation density results from an exact balance between creation and annihilation mechanisms. The PSB walls contain a large density of short edge segments, mostly in the primary plane but also in the cross-slip plane. The microstructure was found to be rather complex [5] and, as yet, former models for saturation in the walls were not revisited. The walls are partly permeable to edge dislocations, but screw dislocation motion in the channels is producing the largest part of the imposed plastic strain amplitude.

In its simplest form, the similitude relation expresses that the characteristic dimension of a dislocation microstructure, $\lambda$, is proportional to the average length of the dislocation segments, $\bar{\ell}$, whereas the flow stress $\tau_{\square}$ is inversely proportional to it. Thus, the flow stress is inversely proportional to the characteristic dimension and is written $\tau_{\square}=K \mu b / \lambda$, where $K$ is a 
similitude constant, $\mu$ is the shear modulus and $b$ is the magnitude of the Burgers vector [7].

The saturation stress of PSBs is strongly temperature dependent, so that investigations on similitude require measurements performed on microstructures formed at different temperatures.

In the recent study [8], the similitude properties of PSB channels and their consequences were investigated in full detail from experimental results in copper and nickel crystals cycled at saturation. The domains of temperature investigated encompassed the whole domain of occurrence of PSBs. It was found that both PSB channels and PSBs follow similitude relations. This may seem paradoxical in the case of PSBs since the wall-widths do not depend on temperature; it is so because of a compensating term arising from PSB channels, in which the critical stress for screw dipole annihilations is smaller than the saturation stress (see the discussion in Section 3 of [8]). The obtained results were confronted to the predictions of Brown's bowing and passing model [9], according to which the critical flow stress of PSB channels is governed by the annihilation of screw dipoles by cross-slip. This critical stress is defined as the stress corresponding to the maximum height of stable dipoles, that is, to their passing stress. As a whole, the predictions of the bowing and passing model were found to be in reasonable agreement with experimental results. However, as the model does not account for any other interaction than the ones between screw dislocations, the critical stresses in the channels were found smaller than the saturation stresses by a few MPa.

An alternative model, the composite model, was developed over the years by Mughrabi and co-workers, essentially at room temperature (see [4] for a full review). This model emphasizes the occurrence of significant internal stresses in the walls and channels and assumes that the annihilations of screw dipoles occurs spontaneously without the help of thermal activation. 
Till now, debates about the modeling of the saturation stress essentially focused on the properties of screw dislocations in PSB channels at $300 \mathrm{~K}$. The motivation of the present work is to provide an extension to a wide range of temperatures. The most basic equation of the bowing and passing model [9] is rather intricate and full predictions cannot be expressed in analytical form. Thus, the model was cast into a more transparent form with the help of a few simplifying assumptions, which were validated at room temperature. In the present work, the basic equation is solved numerically. This makes it possible to predict without any approximation the values of all the relevant quantities as a function of flow stress and temperature. For this purpose, use is made of the results drawn in [8] from experimental studies on copper and nickel.

In Section 2, the basic equations of the model are set in dimensionless forms, which incorporate the similitude constants of PSB channels determined in [8]. The results presented in Section 3 are concerned with the temperature and critical stress dependencies of the critical screw dipole heights and of the coefficient determining the respective contributions of the bowing and passing stresses to the critical stress. Except for the small differences between critical and saturation stresses mentioned above, a striking agreement is found between the model predictions and experimental results on copper and nickel. A few other predictions, in particular on the intrinsic plastic shear amplitudes of PSBs, are discussed in Section 4 and concluding remarks highlighting the major results are presented in Section 5.

\section{The bowing and passing model revisited}

This part recalls first the original bowing and passing model and next the way it is expanded to a wide range of temperatures. Further, a revisited version is established in order 
to verify whether or not an approximation made at room temperature is valid at all temperatures.

\subsection{The original bowing and passing model}

In its original form, Brown's bowing and passing model [9] has brought an improved analytical answer to comments by Mughrabi and Pschenitzka [10] on a previous attempt [11] to estimate the saturation stress in PSB channels.

The discussion of the model is based on Eq. $\left(10^{*}\right)^{1}$. This equation yields the critical stress of the channels $\tau_{c}$ as a linear combination of the Orowan stress $\tau_{\mathrm{Or}}$, which sets screw dislocations in motion, and the passing stress, $\tau_{\text {pass }}$, which corresponds to the maximum critical height, $h_{c}$, for the annihilation of screw dipoles

$$
\tau_{c}=\alpha_{\mathrm{B}} \tau_{\text {Or }}+\tau_{\text {pass }}=\alpha_{\mathrm{B}} \frac{2 E_{\text {edge }}}{b d_{c h}}+\frac{\mu b}{4 \pi h_{c}}
$$

In the assumed absence of any other stress contribution, the flow stress $\tau_{c}$ is assumed to be the stress at saturation. The constant coefficient $\alpha_{\mathrm{B}}$ accounts for the contribution of the Orowan stress to the flow stress. In this Orowan stress, $E_{\text {edge }}$ is the line energy of edge segments (see Supplementary Section S1 for line tensions and line energies) trailed by the bowing screws in channels of width $d_{c h}{ }^{2}$. The contribution of the passing stress is expressed in terms of the critical dipole height, $h_{c}$. In the present context, it is convenient to introduce similitude in Eq. (1) by rewriting it in the form

\footnotetext{
${ }^{1}$ In what follows, starred equations are those of the original bowing and passing model [9].

${ }^{2}$ In Brown's model [9], the channel widths (or wall spacings) are denoted by $d$. In what follows, $d$ denotes the periodicity of a PSB, $d=d_{c h}+d_{w}$, where $d_{w}$ is the wall thickness.
} 


$$
K_{c h}=\frac{\tau_{c} d_{c h}}{\mu b}=\frac{2 \alpha_{\mathrm{B}} E_{\text {edge }}}{\mu b^{2}}+\frac{1}{4 \pi}\left(\frac{d_{c h}}{h_{c}}\right),
$$

where the first equality expresses the similitude relation in the channels and $K_{c h}$ is the related similitude slope. In Eqs. (1) or (2) the channel widths and wall thicknesses are known from experiment. The value usually quoted in the literature for the critical annihilation distance of copper at room temperature is $h_{c} \approx 50 \mathrm{~nm}$ for copper at room temperature [1,12]. The value of the coefficient $\alpha_{\mathrm{B}}$ is critical too because it contributes to the magnitude of resistive stresses in the channels, $\delta \tau_{c h}=\tau_{\mathrm{PSB}}-\tau_{c}$, where $\tau_{\mathrm{PSB}}$ is the saturation stress. Such resistive stresses were found in the previous study [8] but, as they are not included in the bowing and passing model, they do not appear in its equations.

In the model, the coefficient $\alpha_{\mathrm{B}}$ is drawn from a rather complex master equation describing the critical configuration of a dipole of flexible screw dislocations at the passing stress. For this purpose, a small transition zone is defined close to the PSB walls. It connects the straight edge lines deposited on the walls by the motion of the screws to the two interacting screw lines with large critical radii in the central part of the channels. In the transition zone, the characters of the interacting lines go from nearly edge to nearly screw and the curvature radii are quite small. The equilibrium condition for this critical configuration is estimated using an effective value of the line tension, $T_{\text {eff }}$, which is taken for line orientations of $45^{\circ}$ (see Sections S1 and S2.2). As a result, one obtains the master equation given by Eq. $(9 *)$

$$
\tau_{c}=\frac{2 E_{\text {edge }}}{b d_{c h}}+\frac{\mu b}{4 \pi h_{c}}\left(1-\frac{b^{2} d_{c h}^{4}\left(\tau_{c}-\frac{\mu b}{4 \pi h_{c}}\right)^{2}}{640 h_{c}^{2} T_{\text {screw }}^{2}}\right) k
$$


where $k=\left(1-2 T_{\text {eff }} / \tau_{c} d_{c h} b\right)$ and $T_{\text {screw }}$ is the line tension of screw dislocations (Eqs. S1-2 and $\mathrm{S} 2-3)$. At room temperature $k$ does not differ much from unity, so that it is assumed that $k=1$ at all temperatures. Eq. (3) is then rewritten in terms of the coefficient $\alpha_{\mathrm{B}}$ as given by Eqs. (1) or (2). This leads to Eq. $(11 *)$

$$
1-\alpha_{\mathrm{B}}-\alpha_{\mathrm{B}}^{2} \frac{\mu b^{2}}{2 E_{\text {edge }}} \frac{1}{640 \pi}\left(\frac{d_{c h}}{h_{c}}\right)^{3}\left(\frac{E_{\text {edge }}}{T_{\text {screw }}}\right)^{2}=0
$$

The derivation of Eq. (4) is reproduced at end of Section S2.2. The value of $\alpha_{\mathrm{B}}$ is

obtained by introducing into Eq. (4) a plausible value $d_{c h} / h_{c} \approx 30$ in copper at $300 \mathrm{~K}$. This yields $\alpha_{\mathrm{B}} \approx 0.5$, which is adopted for further developments of the original model. According to Eq. (1), the internal stresses calculated by Mughrabi and Pschenitzka [10] should then correspond to a smaller value of $\alpha_{\mathrm{B}}$.

\subsection{The revisited bowing and passing model}

In the original model, Eq. (3) is simplified by taking $k=1$. The equations of the revisited model are obtained by fully treating the term $k$ and solving numerically Eq. (3) for the critical dipole height $h_{c}$. This allows Eq. (4) to be further solved for the coefficient $\alpha_{\mathrm{B}}$. For this purpose, and as was done for Eq. (1), Eqs. (3) and (4) are rewritten in such a way as to incorporate the similitude coefficient of the channels, $K_{c h}$. The expanded forms of Eqs. (3) and (4) are derived in Section S2.2. The revisited form of Eq. (3) is written

$$
F\left(\frac{d_{c h}}{h_{c}}\right)=0=-K_{c h}+\frac{k}{4 \pi}\left(\frac{d_{c h}}{h_{c}}\right)+\frac{2 E_{\text {edge }}}{\mu b^{2}}-k\left(\frac{d_{c h}}{h_{c}}\right)^{3} \frac{\left(K_{c h}-\frac{1}{4 \pi}\left(\frac{d_{c h}}{h_{c}}\right)\right)^{2}}{4 \pi 640\left(T_{\text {screw }} / \mu b^{2}\right)^{2}}
$$


This polynomial equation of fifth order in $d_{c h} / h_{c}$ has a single-valued solution in the domain of temperatures where PSBs are observed. Hence, the temperature dependence of $h_{c}$ can be determined unequivocally. The revisited Eq. (4) is written

$$
\left(1-\alpha_{\mathrm{B}}\right)-\alpha_{\mathrm{B}}^{2} \frac{\mu b^{2}}{2 E_{\text {edge }}} \frac{k}{640 \pi}\left(\frac{d_{c h}}{h_{c}}\right)^{3}\left(\frac{E_{\text {edge }}}{T_{\text {screw }}}\right)^{2}-\frac{(1-k) \mu b^{2}}{8 \pi E_{\text {edge }}}\left(\frac{d_{c h}}{h_{c}}\right)=0
$$

Equation (6) has a single positive root in the interval $0 \leq \alpha_{\mathrm{B}} \leq 1$. At this step, the critical stress in the channels can be obtained directly from Eq. (2) and other properties can be determined by further developing the bowing and passing model. As shown in Section S2.2, setting $k=1$ into Eq. (5) yields Eq. (3) of the original model. One can also easily verify that Eq. (6) reduces to Eq. (4) of the original model.

\section{The expanded bowing and passing model: numerical results}

This part reports on the predictions drawn from the original and revisited models expanded as a function of temperature. The experimental data on $\mathrm{Cu}$ and $\mathrm{Ni}$ are recalled first; they provide input values for solving the two models. The quantities successively determined are the critical dipole heights $h_{c}$, the coefficient $\alpha_{\mathrm{B}}$, the critical stresses $\tau_{c}$ and the dependence of critical dipole heights on critical stresses. All computations were performed using Mathematica v. 6 (Wolfram Research).

\subsection{Experimental data}


The experimental data used in the present study are taken from reference [8], which can be consulted for more detail. For copper, two sets of experimental measurements were lumped together, one by Basinski and Basinski [3] between 4.2 and $295 \mathrm{~K}$ and the other by Holzwarth and Essmann [13,14] between 77 and $430 \mathrm{~K}$. The highest investigated temperatures correspond to the vanishing of the saturation plateau. In what follows, these two data sets are referred to as $\mathrm{Cu}_{\mathrm{BB}}$ and $\mathrm{Cu}_{\mathrm{HE}}$. Although the channel widths exhibit large fluctuations [14], they could be measured with a reasonably good accuracy. The wall thicknesses are constant with $d_{w}=0.11 \pm 0.01 \mu \mathrm{m}$ and, as they were not measured in [3], it was assumed that the value of $d_{w}$ is unchanged below $77 \mathrm{~K}$.

In nickel, the measurements by Tippelt et al. [5] and Hähner et al. [15] include four data points between 77 to $600 \mathrm{~K}$. A data point at $750 \mathrm{~K}$ was removed in [8] because of the coexistence of dislocation cells and PSBs in the microstructure. The wall thicknesses are also constant in nickel with $d_{w}=0.155 \mu \mathrm{m} \pm 10 \%$ [6], but no scatter is available for the channel widths .

The additional data given in Table 1 are drawn from [8] and will be used in what follows. One may notice, as was done in [8], that the similitude properties and the saturation stresses at $0 \mathrm{~K}$ of $\mathrm{Cu}$ and $\mathrm{Ni}$ differ from each other.

\section{Table 1 about here}

The similitude properties of PSBs as a whole were drawn by plotting $\tau_{\mathrm{PSB}} / \mu$ as a function of $b / d$ and taking the slope of the regression line $K_{\mathrm{PSB}}=\tau_{\mathrm{PSB}} d / \mu \square$. The corresponding determination coefficients were always found larger than $R^{2}=0.99$. For $\mathrm{Cu}$ and $\mathrm{Ni}$, the regression lines pass through the origin within quite small stress intercepts,

respectively, $-5 \times 10^{-2} \mathrm{MPa}$ and $1.02 \mathrm{MPa}$. As far as the channels are concerned, the situation is 
different as the stress intercepts are much larger (Table 1). The positive values indicate that the flow stresses of the channels are smaller than the saturation stresses. Similar stress intercepts were measured by Bretschneider et al. [16] in Ni and drawn from several experimental data by Brown (see Section 4 of [9]).

As previously done in [8], all the numerical calculations presented in what follows employ the approximation proposed by Scattergood and Bacon for the shear moduli and Poisson's ratios $(v)$ of $\mathrm{Cu}$ and $\mathrm{Ni}$ (Section $\mathrm{S} 1)$. The temperature dependence of the elastic stiffnesses was taken into account (Section S3). In what follows, the main source of error arises from the measurements of channel widths. The measurements on $\mathrm{Cu}_{\mathrm{HE}}$ are more accurate $( \pm 4 \%)$ than the ones on $\mathrm{Cu}_{\mathrm{BB}}( \pm 7 \%)$. The error on wall thicknesses is comparatively smaller $^{3}$ but it is included.

\subsection{The critical dipole heights in the channels}

The ratio $d_{c h} / h_{c}$ was calculated from the revisited Eq. (5), in which the input quantities are the similitude slope $K_{c h}$ of the channels, the channel widths $d_{c h}$ and the wall thicknesses. The values at $T=0 \mathrm{~K}$ were included. The critical dipole heights were directly obtained from the product $h_{c}=d_{c h}\left(d_{c h} / h_{c}\right)^{-1}$. According to the original model, the ratio $d_{c h} / h_{c}$ should be about 30 or perhaps a bit less. In Table 2, the values of the coefficient $k$, the ratio $d_{c h} / h_{c}$ and the critical dipole heights obtained from the revisited and original versions of the model are compared at three temperatures.

\section{Table 2 about here}

\footnotetext{
${ }^{3}$ The wall thickness only appears in the logarithmic term of the line energy of edge dislocations (Eq. S1-1).
} 
In the revisited model, the values of the coefficient $k$ decrease in a non-negligible manner with increasing temperature. Hence, it seems that the approximation $k=1$ is rather rough. Whereas the original model yields ratios $d_{c h} / h_{c}$ that decrease with increasing temperature, these ratios are almost constant in the revisited model. This arises from a compensating effect of the term $k$. In the revisited model, the critical screw dipole heights at room temperature are close to the most frequently quoted value of $50 \mathrm{~nm}$ for copper [1,12], specifically $44.8 \pm 3 \mathrm{~nm}$ for a saturation stress of $29 \mathrm{MPa}$ in $\mathrm{Cu}_{\mathrm{BB}}$ and $49.3 \pm 2 \mathrm{~nm}$ for a saturation stress of $28 \mathrm{MPa}$ in $\mathrm{Cu}_{\mathrm{HE}}$. For nickel $h_{\mathrm{c}}(293 \mathrm{~K}) \approx 48 \mathrm{~nm}$.

Figure 1a shows revisited similitude plots of the scaled saturation stresses as a function of the scaled critical dipole heights in $\mathrm{Cu}$ and $\mathrm{Ni}$. The similitude slopes are almost the same and the positive stress intercepts reveal the occurrence of resistive stresses, which will be further discussed in Section 3.3. Fig. 1b shows the temperature dependence of the revisited critical dipole heights.

\section{Figure lab about here}

One can see from overlapping data in Fig. 1b that the dipole heights are almost identical in $\mathrm{Cu}$ and Ni. The only significant differences reside in the critical stresses at $0 \mathrm{~K}$ (Table 1) and in the maximum temperatures at which a saturation plateau was obtained. The same features were experimentally observed on the temperature dependence of the scaled stresses $\tau_{\text {III }}$ in monotonic deformation (see [17] p. 160 and Fig. 28 of [6]). Hence, in spite of their similarities in an intermediate temperature domain, the stress dependences of the activation energies for screw dipole annihilations in $\mathrm{Cu}$ and $\mathrm{Ni}$ are expected to be different. This is not surprising since the scaled stacking fault energies $\gamma / \mu b$ are also different, $4.0 \times 10^{3}$ for $\mathrm{Cu}$ and $6.0 \times 10^{3}$ for Ni (see Table B1 of [18]). 
In copper, the critical dipole heights at $0 \mathrm{~K}$ are $h_{c o}(\mathrm{Cu}) \approx 16 \mathrm{~nm}$ and $h_{c o}(\mathrm{Ni}) \approx 20 \mathrm{~nm}$. The simulations by Pauš et al. [19] shown in Fig. 1b yield similar values for $\mathrm{Cu}$ at all temperatures, with $h_{c}(\mathrm{Cu})=23 \mathrm{~nm}$ at $4.2 \mathrm{~K}$. Similar values were also found for nickel at 77 and $300 \mathrm{~K}$ (see Table 1 of [19]). Mitchell [17] also compiled extrapolated resolved stresses for the onset of the dynamic recovery stage at $0 \mathrm{~K}$ in monotonic deformation ${ }^{4}$. From his data and the similitude relations of the critical dipole heights (Fig. 1a), one draws $h_{c o}(\mathrm{Cu})=9-17$ $\mathrm{nm}$ and $h_{c \mathrm{o}}(\mathrm{Ni})=12-35 \mathrm{~nm}$. These comparisons show that the present results are compatible with experiment and confirm the thermally activated character of screw dipole annihilations.

Although atomistic simulations also confirmed that screw dislocation cross-slip is thermally activated [20], only few of them were devoted to the annihilation of screw dipoles (e.g., [20-22]). Unfortunately, they did not incorporate the contribution of the externally applied stress, so that the critical dipole heights at $0 \mathrm{~K}$ were too small and the critical stresses too high. In such stress-free conditions, it is not possible to obtain a realistic insight into the bowing and passing mechanism.

\subsection{The coefficient $\alpha_{\mathrm{B}}$ and the critical stresses in the channels}

As the values of $k$ and $d_{c h} / h_{c}$ are known, the coefficient $\alpha_{\mathrm{B}}$ can be calculated from Eqs. (4) and (6) for the two versions of the expanded model. The results are given in Table 3.

\section{Table 3 about here}

The original model yields $\alpha_{\mathrm{B}}$ values that increase with increasing temperature. The stabilizing effect of the term $k$ manifests itself again in the revisited model. In $\mathrm{Cu}$, one has $\alpha_{\mathrm{B}}$

\footnotetext{
${ }^{4}$ In metals with low stacking fault energy, twinning may interfere with dynamic recovery.
} 
$(\mathrm{Cu})=0.503 \pm 0.007$ over the whole temperature range. Hence, the value estimated by Brown

[9] at $300 \mathrm{~K}, \alpha_{\mathrm{B}} \approx 0.5$, is actually temperature-independent. For $\mathrm{Ni}, \alpha_{\mathrm{B}}(\mathrm{Ni})=0.532 \pm 0.04$.

As the values of $d_{c h} / h_{c}$ and $\alpha_{\mathrm{B}}$ are known, the critical stresses $\tau_{c}$ can be directly estimated from Eq. (2). In the two versions of the model, the similitude coefficient of the channels, $K_{c h}$, is an input property. For both $\mathrm{Cu}$ and $\mathrm{Ni}$, the critical stresses are found smaller than the saturation stresses. Hence, the stress difference $\delta \tau_{c h}=\tau_{\mathrm{PSB}}-\tau_{c}$ represents additional resistive stresses. The stresses $\delta \tau_{c h}$ and $\tau_{c}$ yielded by the original model are obtained as follows. The similitude relation in the channels is written $K_{c h}=\tau_{c} d_{c h} / \mu b$ and the resistive stresses are obtained from

$$
\delta \tau_{c h}=\tau_{\mathrm{PSB}}-\tau_{c}=\tau_{\mathrm{PSB}}-\frac{K_{c h} \mu b}{d_{c h}},
$$

where all numerical values are known from experiment in the last term at the right-hand side. The original model yields almost exactly the same results as the comparison with experiment performed in [8]. For instance, the average values, 5.33 $\mathrm{MPa}$ for $\mathrm{Cu}$ and $5.97 \mathrm{MPa}$ for $\mathrm{Ni}$, are very close to the ones previously determined by another method (Table 1). The same holds for the individual resistive stresses. In the revisited model, the term $k$ slightly modifies the similitude relation because it contains a drifting logarithmic term (Eq. S2-3). As a result, the average resistive stresses $\delta \tau_{c h}$ decrease to $5.0 \mathrm{MPa}$ in $\mathrm{Cu}$ and $4.84 \mathrm{MPa}$ in Ni.

All the stresses discussed above, plus the bowing stress, are plotted as a function of temperature in Fig. 2a. The resistive stresses are obtained through a difference between two large quantities. Thus, they exhibit large fluctuations, at least $\pm 8 \%$ for $\mathrm{Cu}_{\mathrm{HE}}$ and $\pm 14 \%$ for $\mathrm{Cu}_{\mathrm{BB}}$, owing to the accumulation of known and unknown experimental uncertainties. In addition, they do not seem to exhibit a well-marked temperature dependence. 
Fig. 2ab about here

Figure $2 \mathrm{~b}$ shows a similitude plot of the critical stresses in the revisited model as a function of the critical dipole heights in $\mathrm{Cu}$ and $\mathrm{Ni}$. The similitude coefficients $\left(K_{h c}\right)$ only slightly differ from each other and from the ones obtained for the whole PSBs in the revisited model (Fig. 1a). The stress intercepts are negligibly small and well below experimental uncertainties. The similitude properties of the channels in the revisited model are only slightly modified with respect to the ones previously obtained in Ref. [8]. For $\mathrm{Cu}$ and Ni, the coefficients $K_{h c}=\tau_{c \text {, rev. }} d_{c h} / \mu b$ respectively differ by $2 \%$ and $0.5 \%$.

In summary the revisited model is more accurate than the original one. It yields constant values for the coefficient $\alpha_{\mathrm{B}}$ and the ratio $d_{c h} / h_{c}$ as well as critical stresses in the channels that are smaller than the saturation stresses by a few MPa. The critical screw dipole heights are thermally activated, they follow a similitude relation and their values at $300 \mathrm{~K}$ is $h_{c} \approx 50 \mathrm{~nm}$.

\section{Additional predictions of the revisited bowing and passing model}

Brown's model [9] contains a few predictions for persistent slip, which do not derive directly from the complex Eq. (5) and are drawn from various other considerations.

\subsection{Equilibrium of PSB walls and similitude}

The basic Eq. (1) is rewritten in Eq. $\left(12^{*}\right)$ as a sum of the Orowan stress and a complementary passing stress term $\tau_{\mathrm{p}}$ 


$$
\tau_{c}=\tau_{\mathrm{Or}}+\tau_{p}=\tau_{\mathrm{Or}}+\left(\frac{\mu b}{4 \pi h_{c}}-\left(1-\alpha_{\mathrm{B}}\right) \tau_{\mathrm{Or}}\right)
$$

The original model [9] is further developed on the basis of Eq. (8) by making an hypothesis about the equilibrium of PSB walls. Considering that the motion of screws under the Orowan stress $\tau_{\text {Or }}$ tends to push too closely spaced walls back in place, whereas the formation of screw dipoles tends to pull the walls together, the equilibrium of wall spacings requires that $\tau_{\mathrm{Or}} / \tau_{p}=1$ or, equivalently, $\tau_{c}=2 \tau_{\mathrm{Or}}$. By importing these two relations in Eq. (8) one obtains Eq. $\left(14^{*}\right)$ of the original model, $\tau_{c}=\mu b / 2 \pi\left(2-\alpha_{\mathrm{B}}\right) h_{c} . \quad$ This last equation is rewritten in the the form of a similitude relation

$$
K_{h c}=\frac{\tau_{c} h_{c}}{\mu b}=\frac{2 \tau_{\mathrm{Or}} h_{c}}{\mu b}=\frac{1}{2 \pi\left(2-\alpha_{\mathrm{B}}\right)} \approx \frac{1}{3 \pi}
$$

The relations drawn from the equilibrium hypothesis were tested on the revisited version of the model. For $\mathrm{Cu}$ and $\mathrm{Ni}$, the ratios $\tau_{\mathrm{Or}} / \tau_{p}$ are almost independent of temperature with average values larger than unity, $1.200 \pm 0.003 \mathrm{in} \mathrm{Cu}$ and $1.36 \pm 0.03 \mathrm{in} \mathrm{Ni}$. The ratios $\tau_{c} / \tau_{\mathrm{Or}}$ behave in the same manner with average values smaller than two, $1.83 \pm 0.03$ in $\mathrm{Cu}$ and 1.73 \pm 0.01 in Ni. By reproducing the simple calculation performed above without attributing values to the two ratios and to $\alpha_{\mathrm{B}}$, one obtains the following corrected form of Eq. (9)

$$
K_{h c}=\frac{\tau_{c} h_{c}}{\mu b}=\frac{\left(\tau_{c} / \tau_{\mathrm{Or}}\right) \tau_{\mathrm{Or}} h_{c}}{\mu b}=\frac{\left(\tau_{c} / \tau_{\mathrm{Or}}\right)}{4 \pi\left(1+\left(\tau_{p} / \tau_{\mathrm{Or}}\right)-\alpha_{\mathrm{B}}\right)}=0.109(\mathrm{Cu}) ; 0.114(\mathrm{Ni})
$$

This relation reproduces almost perfectly the two values of the similitude coefficients given in Fig. 2b. Thus, the two stress ratios $\tau_{\mathrm{Or}} / \tau_{p}$ and $\tau_{c} / \tau_{\mathrm{Or}}$ do have constant values. 
The measured channel widths, which are input quantities, are all fixed; in copper they are all given with error bars representing the amplitude of their fluctuations. Eq. (10) shows that in such conditions, the hypothesis based on the equilibrium of the walls is by construction embedded into the bowing and passing model. Thus, no assumption is needed because the constancy of the two stress ratios simply derives from the fact that the critical dipole heights follow a similitude relation.

\subsection{The intrinsic plastic strain amplitude of PSBS}

The intrinsic plastic strain amplitude of PSBs is defined as the shear strain $\gamma_{\text {PSB }}$ per quarter of cycle under the saturation stress $\tau_{\mathrm{PSB}}$. Reliable experimental values of this quantity are rather scarce. Here, use is made of the data by Holzwarth and Essmann [14] in copper, which agree well with other published values. The plastic strain amplitude was found to depend linearly on the saturation stress. Four values of $\gamma_{\text {РsB }}$ were determined at different temperatures from direct measurements of the active volume fraction of PSBs.

The plastic strain amplitude $\gamma_{\text {PSB }}$ was estimated by Brown in 2004 [11]. The probability for complete annihilations of screw dislocations was calculated as a function of the probability $p$ that an edge dislocation crosses a wall (Eq. (20) of [11]). In the improved model [9], the introduction of the coefficient $\alpha_{\mathrm{B}}$ does not modify the previous result. As all experimental results were plotted as a function of $\tau_{\mathrm{PSB}}$ the critical stress $\tau_{\mathrm{c}}$ (Eq. (15*) of [9]) is replaced here by $\tau_{\mathrm{PSB}}$. A linear relation in then obtained between $\gamma_{\mathrm{PSB}}$ and $\tau_{\mathrm{PSB}}$

$$
\gamma_{\mathrm{PSB}}=\frac{\pi(1+p)^{2}}{(1-p)^{2}} \frac{3 d_{c h}}{3 d_{c h}+d_{w}} \frac{\tau_{\mathrm{PSB}}}{\mu}=4 \pi \frac{3 d_{c h}}{3 d_{c h}+d_{w}} \frac{\tau_{\mathrm{PSB}}}{\mu} \approx 4 \pi \frac{\tau_{\mathrm{PSB}}}{\mu}
$$


It was shown in [11] that $p \approx 1 / 3$, so that the term containing the probability $p$ is equal to $4 \pi$. The next term is a tentative correction close to unity, which accounts for screw dipole annihilations in the walls. The prediction drawn from Eq. (11) holds for the two versions of the model since the involved quantities are the same in both cases. It is compared to the experimental results on $\mathrm{Cu}_{\mathrm{HE}}$ in Fig. 3a. The experimental and predicted linear dependences on saturation stress are somehow different. An attempt was made to fit the experimental data by adjusting the value of the coefficient $p$. The best result was obtained by taking $p=0.39$.

\section{Fig. 3ab about here}

Brown [11] proposed a simplified alternative model for $\gamma_{\mathrm{PSB}}$ based on the annihilations of rectangular edge-screw loops expanding through walls and channels (see Section S4 for more detail on the model). The intrinsic plastic shear strain amplitude of PSBs is given by

$$
\gamma_{\mathrm{PSB}}=b / 2 F h_{c},
$$

where $F$ is a free statistical parameter. With $F=1.43$, Eq. (12) reproduces rather well the experimental results (Fig. 3b).

Figure 3 shows that the predictive ability of Eqs. (11) and (12) may be improved by adjusting in each case the value of a single coefficient. In the two equations it is assumed that the channels and walls deform under an uniform saturation stress. This cannot be since the flow stress of the channels is smaller than the saturation stress, so that the flow stress of the walls is necessarily larger than $\tau_{\mathrm{PSB}}$. Furthermore, the real microstructure of the walls that was described by Tippelt et al. [5] is much more complex than the traditional ones that were used to derive Eqs. (11) and (12). In particular the walls contain a large density of small prismatic dislocation loops lying in the cross-slip plane, which should strongly interact with incoming 
dislocations. Thus, the loops expanding through the walls must exhibit quite complicated shapes.

\section{Concluding remarks}

The bowing and passing model for PSBs [9] was extended to the whole domain of temperatures in which a saturation plateau is observed. Advantage was taken of the similitude relation to rewrite in dimensionless form the equations of the original model and of a revisited model, which is more accurate. Input quantities for the computation of solutions were taken from a previous study of experimental results on copper and nickel. In such conditions, all unknown coefficients and variables were directly calculated without any assumption or approximation.

The comparison between experimental results and the predictions of the original and revisited bowing and passing models were discussed in the previous sections. This part emphasizes the major results obtained from the revisited model and their consequences.

- Two important quantities were found to be constant irrespective of temperature. i) - The ratio $d_{c h} / h_{c}$ of the channel width to the critical dipole height is about $29 \mathrm{in} \mathrm{Cu}$, close to the value assumed by Brown [9] at $300 \mathrm{~K}$, and about 25 in Ni. ii) - The coefficient $\alpha_{\mathrm{B}}$ governs the contribution of the Orowan stress to the critical stress and the magnitude of the resistive stress in the channels. Its values are $\alpha_{\mathrm{B}}(\mathrm{Cu})=0.5$, as was assumed by Brown [9] at $300 \mathrm{~K}$, and $\alpha_{\mathrm{B}}(\mathrm{Ni})=0.53$.

- The critical dipole heights, $h_{c}$, were computed as a function of temperature in the whole domain of existence of PSBs. At room temperature, the revisited model yields values close to $50 \mathrm{~nm}$ in copper, in agreement with the experimental estimate given in [1,12], and in nickel. At $0 \mathrm{~K}$, the critical dipole heights are $h_{c o}(\mathrm{Cu})=16 \mathrm{~nm}$ and $h_{c o}(\mathrm{Ni})=20 \mathrm{~nm}$. Although 
atomistic simulations confirmed that cross-slip is a thermally activated phenomenon, they did not provide realistic predictions for the critical dipole heights. The present results may serve as a benchmark test for further studies, in which an external loading is applied to the interacting screw dislocations.

- The critical dipole heights are related to the critical stresses in the channels, $\tau_{c}$, through a perfect similitude relation passing through the origin (Fig. 2b). The channels widths are also related to the critical stresses through another perfect similitude relation (Fig. 6 of [8]). This arises because the ratio $d_{c h} / h_{c}$ is a constant. The ratio $\tau_{c} / \tau_{\mathrm{Or}}$ of the critical stress to the Orowan stress in the channels was assumed in [9] to be a constant from considerations on the equilibrium of the walls. The value of this ratio is actually rooted in the similitude relation between the critical dipole heights and the critical stresses (Section 3.1). Finally, the critical stresses at $0 \mathrm{~K}$ are similar to the critical stresses for the onset of the dynamic recovery stage III in monotonic deformation (Section 3.2). These results are of prime importance because they confirm again the basic assumption of the bowing and passing model, according to which the thermally activated annihilation of screw dipoles is governing the channel widths, the Orowan stresses and the critical stresses in the channels. In contrast, the central role played by the critical dipole height challenges a basic assumption of the composite model according to which the annihilation of screw dipoles is only stress-assisted.

- In this study no new information could be drawn about the origin of the small resistive stresses in the channels. It is only confirmed that the critical stresses in the channels are slightly smaller than the saturation stresses by a few MPa and that they do not exhibit a wellmarked temperature dependence [8]. In this previous study the critical stresses were directly obtained from experiment through the stress intercepts of the similitude relation in the channels. It was shown in Section 3.1 of [8] that the use of the line tension proposed by Scattergod and Bacon [S3] would slightly improve the results. Nevertheless, the magnitudes 
of the resistive stresses in the channels are now reasonably well know. Their existence was not considered in the bowing and passing model; taking them into account allowed all other predictions of the model to be confirmed. According to Mughrabi and Pschnenitzka [10], the critical stress $\tau_{c}$ should be about $20 \%$ larger than the largest of the contributions from the Orowan and dipole bowing stresses. In copper at room temperature, we obtained from the present data a resistive stress of $11 \mathrm{MPa}$, whereas the revisited model yields a resistive stress around $4 \mathrm{MPa}$, and a coefficient $\alpha_{\mathrm{B}} \approx 0.2$ instead of 0.5 . Thus the model [10] overestimates the resistive stresses.

- The first dislocation dynamics simulations on channels of persistent slip bands [23-25] were intended to compare the predictions of Brown's original model [9] and the model by Mughrabi and Pschnenitzka [10]. No real consensus was obtained because these simulations, as well as the model, strongly depended on simplifying assumptions made about the complex microstructure of the walls. More recent DD simulations (e.g. [19]) and mass simulations that are now in progress will certainly contribute to clarify the situation.

- The models proposed by Brown $[9,11]$ for the intrinsic plastic strain amplitude of PSBs take into account the penetration and crossing of PSB walls by edge dislocations. However, to obtain accurate predictions, it is necessary to account in a more realistic manner for the microstructure of the walls. This modeling task should be performed in parallel with dislocation dynamics simulations and atomistic simulations for short-range interactions.

In summary, the numerical solutions of Brown's expanded and revisited model for bowing and passing [9] are in excellent agreement with its basic assumptions and predictions. The only exception is the occurrence of small resistive stresses in the channels. In addition, much remains to be understood about the behavior of the walls. Analytical approaches may be extremely complex and only mesoscale mass simulations and atomistic simulations can help 
going further. Finally, it may be interesting to examine as a function of temperature the compatibility of the composite model [4] with the bowing and passing model.

\section{Appendix A. Supplementary data}

Supplementary data associated with this article can be found at 


\section{References}

[1] H. Mughrabi, F. Ackermann, K. Herz, Persistent slip bands in fatigued face-centered and body-centered metals. In: J.T. Fong (Ed.), Fatigue Mechanisms, ASTM-STP 675, Philadelphia (PA), 1979, pp. 69-105.

[2] L.M. Brown, Dislocations and the fatigue strength of metals. In: M.F. Ashby, R.

Bullough, C. Hartley and J.P. Hirth (Eds.), Dislocation Modelling of Physical Systems, Pergamon Press, New York, 1981, pp. 51-68.

[3] Z.S. Basinski, S.J. Basinski, Fundamental aspects of low amplitude cyclic deformation in face-centred cubic crystals, Progr. Mater. Sci. 36 (1992) 89-148.

[4] H. Mughrabi, T. Ungár, Long-range internal stresses in deformed single-phase materials: The composite model and its consequences. In: F.R.N. Nabarro and M.S. Duesbery (Eds.), Dislocations in Solids, Vol. 11, Elsevier Science B.V., Amsterdam, 2002, pp. 343-411.

[5] B. Tippelt, J. Bretschneider, P. Hähner, The dislocation microstructure of cyclically deformed nickel single crystals at different temperatures, Phys. Stat. Sol. (a) 163 (1997) 1126.

[6] M. Sauzay, L. Kubin, Scaling laws for dislocation microstructures in monotonic and cyclic deformation of fcc metals. Progr. Mater. Sci. 56 (2011) 725-784.

[7] D. Kuhlmann-Wilsdorf, A new theory of work hardening, Trans. Metall. Soc. AIME 224 (1962) 1047-1061.

[8] L. Kubin, M. Sauzay, Persistent slip bands: Similitude and its consequences, Acta Mater., 104 (2016) 295-302.

[9] L.M. Brown, Dislocation bowing and passing in persistent slip bands, Phil. Mag. 86 (2006) 4055-4068.

[10] H. Mughrabi, F. Pschenitzka, Constrained glide and interaction of bowed-out screw dislocations in confined channels. Phil. Mag. 85 (2005) 3029-3045. 
[11] L.M. Brown, A discussion on the structure and behaviour of dipole walls in cyclic plasticity. Phil. Mag., 21 (2004) 2501-2520.

[12] U. Essmann, H. Mughrabi, Annihilations of dislocations during tensile and cyclic deformation and limits of dislocation densities, Phil. Mag. 40 (1979) 731-756.

[13] U. Holzwarth, U. Essmann, Transformations of dislocation patterns in fatigued copper crystals, Mater. Sci. Eng. A164 (1993) 206-210.

[14] U. Holzwarth, U. Essmann, Temperature-induced rearrangement of the dislocation pattern of persistent slip bands in copper single crystals, Appl. Phys. A 58 (1994) 197-210. [15] P. Hähner, B. Tippelt, C. Holste, On the dislocation dynamics of persistent slip bands in cyclically deformed F.C.C. metals, Acta Mater. 46 (1998) 5073-5084.

[16] J. Bretschneider, C. Holste, B. Tippelt, Cyclic plasticity of nickel single crystals at elevated temperatures, Acta Mater. 45 (1997) 3775-3783.

[17] T.E. Mitchell, Dislocations and plasticity in single crystals of face-centred cubic metals and alloys, Progr. Appl. Mater. Res. 6 (1964) 117-237.

[18] L.P. Kubin, Dislocations, Mesoscale Simulations and Plastic Flow, Oxford Series on Materials Modelling, A.P. Sutton and R.E. Rudd (Eds.), Oxford University Press, 2013. [19] P. Pauš, J. Kratochvíl, M. Beneš, A dislocation dynamics analysis of the critical crossslip annihilation distance and the cyclic saturation stress in fcc single crystals at different temperatures, Acta Mater. 61 (2013) 7917-7923.

[20] T. Vegge, K. Jacobsen, Atomistic simulations of dislocation processes in copper, J. Phys.: Condens Matter 14 (2002) 2929-2956.

[21] D. Mordehai, I. Kelson, G. Makov, Cross-slip and annihilation of screw dislocations in Cu: a molecular dynamics study, Mater. Sci. Eng. A 400-401 (2005) 37-39.

[22] S. Rao et al., Screw dislocation cross slip at cross-slip plane jogs and screw dipole annihilations in FCC Cu and Ni investigated via atomistic simulations, Acta Mater. 101 (2015) 10-15. 
[23] K.W. Schwarz and Mughrabi H., Interaction and passing stress of two threading dislocations of opposite sign in a confined channel. Phil. Mag. Lett., 86 (2006) 773-785. [24] J.A. El Awady, N.M. Ghoniem and Mughrabi H., Dislocation modelling of localized plasticity in persistent slip bands. In B.L. Adams and A. Garmestani (Eds.), Mechanics and Materials Modeling and Materials Design Methodologies, $136^{\text {th }}$ Annual Meeting and Exhibition, TMS, Warrendale PA, USA, 2007, pp. 23-35.

[25] J. Křišstan and J. Kratochvíl, Interaction of glide dislocations in a channel of a persistent slip band, Phil. Mag. 87 (2007) 4593-4616. 

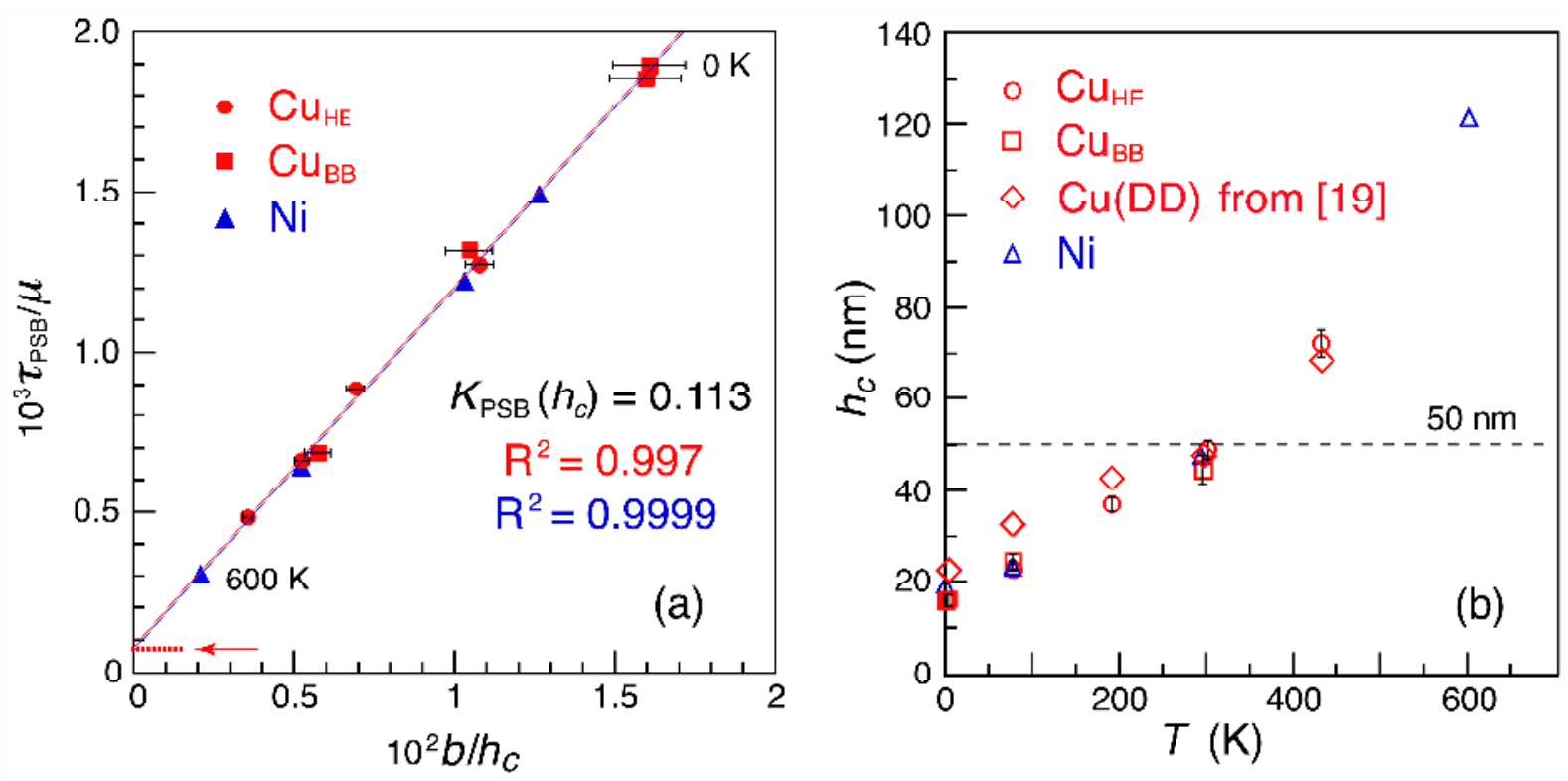

Fig. 1. Critical dipole heights, $h_{c}$, in $\mathrm{Cu}$ and $\mathrm{Ni}$ as yielded by the revisited model. The values at $0 \mathrm{~K}$ and available error bars are included $\left( \pm 8 \%\right.$ for $\mathrm{Cu}_{\mathrm{HE}}$ and $\pm 14 \%$ for $\left.\mathrm{Cu}_{\mathrm{BB}}\right)$. (a) Similitude plot of the scaled saturation stress $\tau_{\mathrm{PSB}} / \mu \mathrm{vs} . h_{c} / b$. The arrow near the origin points at small intercepts with the stress axis. (b) Temperature dependence of the critical dipole heights. The data $\mathrm{Cu}_{\mathrm{DD}}$ is taken from dislocation dynamics simulations of the bowing and passing model performed by Pauš et al. [19]. 

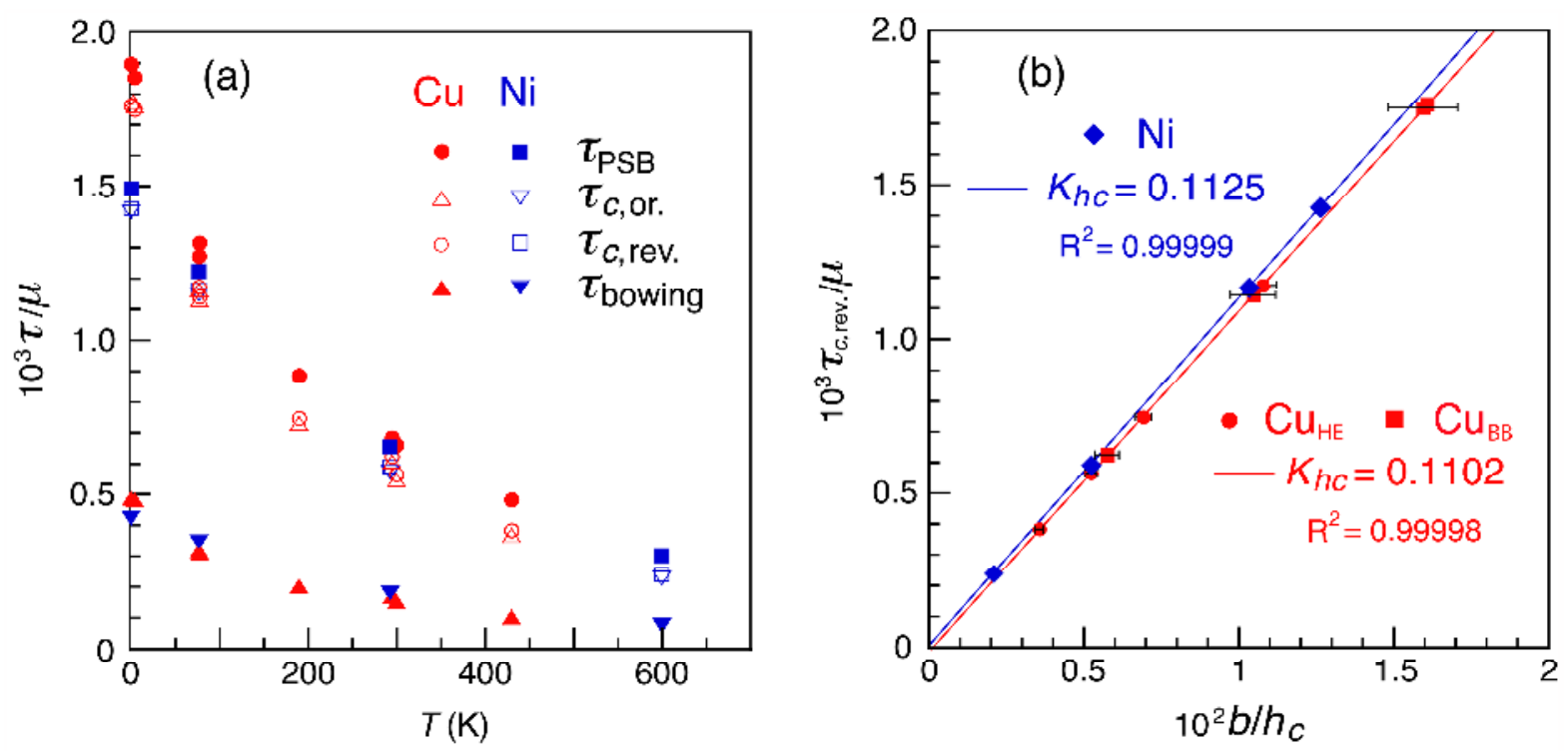

Fig. 2. (a) Temperature dependence of several stresses in $\mathrm{Cu}$ and Ni. $\tau_{\mathrm{PSB}}$ is the saturation

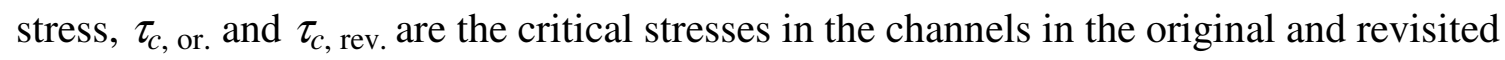
versions of the model and $\tau_{\text {bowing }}$ is the bowing stress in the revisited model. (b) Similitude plot of the scaled critical stresses $\tau_{c \text {, rev. }} / \mu$ as a function of $b / h_{c}$. The stress values at $0 \mathrm{~K}$ are included in both figures. The error bars are $\pm 8 \%$ for $\mathrm{Cu}_{\mathrm{HE}}$ and $\pm 14 \%$ for $\mathrm{Cu}_{\mathrm{BB}}$. 

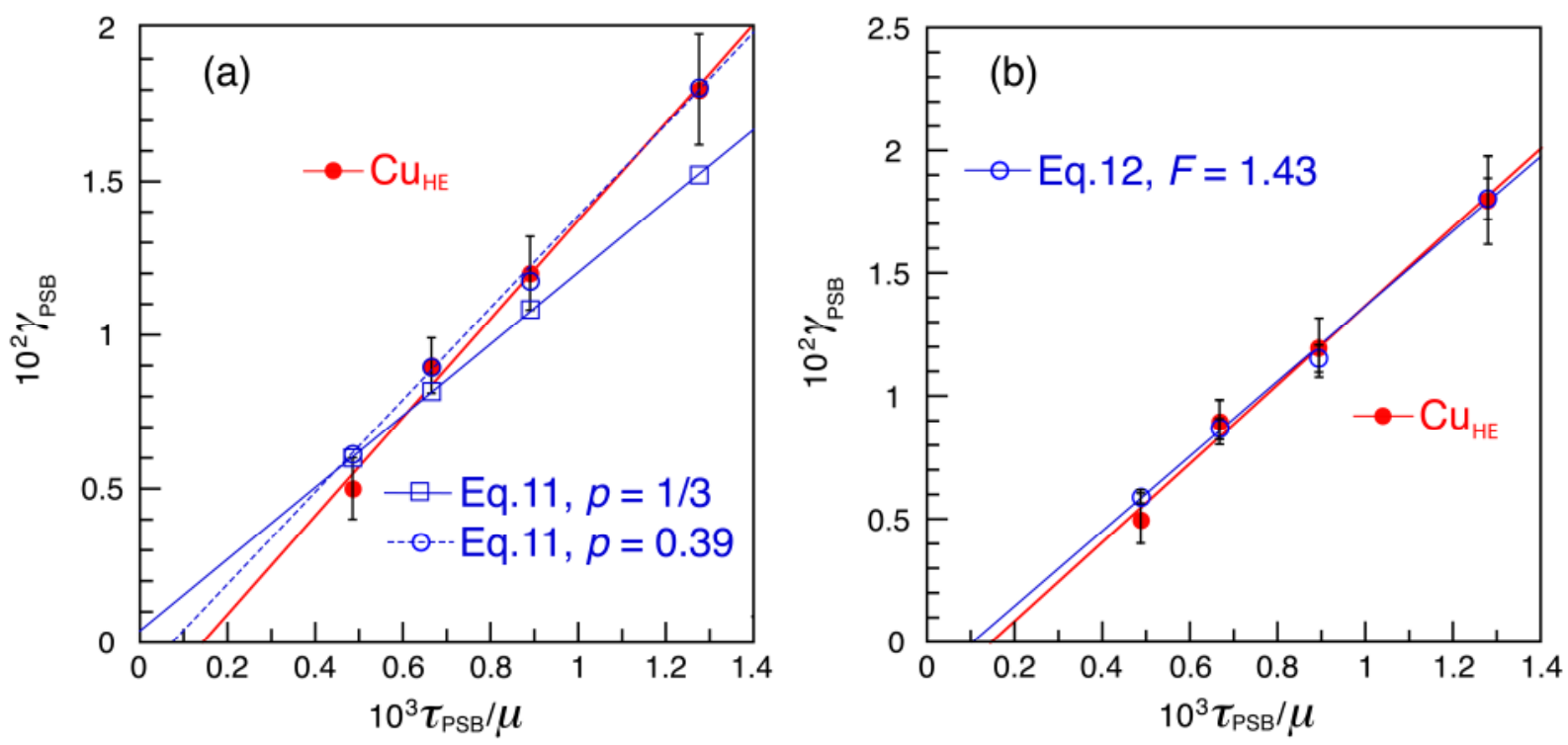

Fig. 3.

The plastic strain amplitude of PSBs, $\gamma_{\mathrm{PSB}}$, as a function of the scaled critical stress $\tau_{\mathrm{PSB}}$ in $\mathrm{Cu}_{\mathrm{HE}}$ (after [14]) compared to the predictions of the bowing and passing model. (a)

Predictions drawn from Eq. (11), with $p=1 / 3$ and from a fit with $p=0.39$. (b) Prediction obtained from Eq. (12) for the revisited model with a value $F=1.43$ for the free-parameter.

The error bars of the measured and predicted values are included. 


\section{Table 1}

Data drawn from experimental results on $\mathrm{Cu}\left(\mathrm{Cu}_{\mathrm{BB}}\right.$ and $\mathrm{Cu}_{\mathrm{HE}}$ merged together) and Ni. From left to right: - the extrapolated saturation stresses at $0 \mathrm{~K}$ scaled by the shear modulus at $0 \mathrm{~K}$, the similitude slopes for PSBs $\left(K_{\mathrm{PSB}}\right)$ and PSB channels $\left(K_{\mathrm{ch}}\right)$ with, in parentheses, the stress intercepts (in MPa) and the correlation coefficients, - the average values of the moderately temperature-dependent resistive stresses in the channels (in $\mathrm{MPa}$ ). The stresses at $0 \mathrm{~K}$ are included in the last three sets of results.

$$
\tau_{\mathrm{o}} / \mu_{\mathrm{O}} \quad K_{\mathrm{PSB}} \quad K_{c h} \quad<\delta \tau_{c h}>
$$

$\mathrm{Cu} \quad 1.9010^{3} \quad 4.06,(-0.05-0.992) \quad 3.10,(5.40-0.992) \quad 5.31$

$\mathrm{Ni} \quad 1.5510^{3} \quad 3.58,(1.02-0.999) \quad 2.78,(5.53-0.998) \quad 5.96$ 


\section{Table 2}

Values of $k, d_{c h} / h_{c}$ and the critical dipole heights $h_{c}$ in $\mathrm{Cu}$ and $\mathrm{Ni}$ at $0 \mathrm{~K}$, room temperature (RT) and the maximum temperature of the saturation plateau $\left(T_{\max } \approx 430 \mathrm{~K}\right.$ for $\mathrm{Cu}$ and $\approx 600$ $\mathrm{K}$ for Ni). The calculations were performed on the expanded original (or.) and revisited (rev.) versions of Brown's model [9]. The room temperature values are $295 \mathrm{~K}$ for $\mathrm{Cu}_{\mathrm{BB}}$ (left), $300 \mathrm{~K}$ for $\mathrm{Cu}_{\mathrm{HE}}$ (right) and $293 \mathrm{~K}$ for $\mathrm{Ni}$.

\begin{tabular}{|c|c|c|c|c|}
\hline$T(\mathrm{~K})$ & 0 & 300 & $T_{\max }$ & \\
\hline$k_{\mathrm{B}}$ & 1 & 1 & 1 & or. \\
\hline$k_{\mathrm{B}}(\mathrm{Cu})$ & 1.0 & $0.94 \quad 0.93$ & $0.90_{5}$ & rev. \\
\hline$k_{\mathrm{B}}(\mathrm{Ni})$ & 0.98 & 0.92 & 0.86 & rev. \\
\hline \multirow{2}{*}{$d_{c h} / h_{c}(\mathrm{Cu})$} & 28.6 & $26.6 \quad 26.4$ & 25.4 & or. \\
\hline & 28 & $29.0 \quad 29.1$ & 29.5 & rev. \\
\hline \multirow{2}{*}{$d_{c h} / h_{c}(\mathrm{Ni})$} & 24 & 23.6 & 18.8 & or. \\
\hline & 24.6 & 25.0 & 24.7 & rev. \\
\hline$h_{c}(\mathrm{Cu}) \mathrm{nm}$ & $15.9_{5}$ & $48.9_{5}$ & 84.1 & rev. \\
\hline$h_{c}(\mathrm{Ni}) \mathrm{nm}$ & 19.8 & 47.9 & 121.5 & rev. \\
\hline
\end{tabular}




\section{Table 3}

Rounded values of $\alpha_{\mathrm{B}}$ in $\mathrm{Cu}$ and $\mathrm{Ni}$ at $0 \mathrm{~K}$, room temperature (RT) and the maximum temperature $\left(T_{\max }=430 \mathrm{~K}\right.$ for $\mathrm{Cu}$ and $600 \mathrm{~K}$ for $\mathrm{Ni}$ ) in the original (or.) and revisited (rev.) versions of Brown's model [9]. The room temperature values are $295 \mathrm{~K}$ for $\mathrm{Cu}_{\mathrm{BB}}$ (left), $300 \mathrm{~K}$ for $\mathrm{Cu}_{\mathrm{HE}}$ (right) and $293 \mathrm{~K}$ for $\mathrm{Ni}$.

\begin{tabular}{cccccc}
$T(\mathrm{~K})$ & 0 & \multicolumn{2}{c}{300} & $T_{\max }$ & \\
\hline$\alpha_{\mathrm{B}}(\mathrm{Cu})$ & 0.49 & 0.57 & 0.58 & 0.62 & or. \\
& 0.50 & 0.51 & 0.50 & 0.51 & rev. \\
\hline$\alpha_{\mathrm{B}}(\mathrm{Ni})$ & 0.53 & 0.63 & 0.74 & or. \\
& 0.52 & 0.53 & 0.56 & rev. \\
\hline
\end{tabular}

